\title{
Nonlinearities in Conservative Growth Equations
}

\author{
Abhijit K. Kshirsagar \\ Raman Research Institute, Bangalore 560080, India \\ S. V. Ghaisas \\ Department of Electronics Science, University of Pune, Pune 41100\%, India
}

\begin{abstract}
Using the dynamic renormalization group (DRG) technique, we analyze general nonlinearities in a conservative nonlinear growth equation with non-conserved gaussian white noise. We show that they fall in two classes only: the Edwards-Wilkinson and Lai-Das Sarma types, by explicitly computing the associated amputated two and three point functions at the first order in perturbation parameter(s). We further generalize this analysis to higher order nonlinearities and also suggest a physically meaningful geometric interpretation of the same.
\end{abstract}

Growth of thin films from vapor has been a subject of significant interest for both experimentalists and theorists. The non-equilibrium nature of this growth makes the techniques applicable to the study of fractals, available for understanding the physics of thin film growth; particularly in the thermodynamic limit [1]. Most of the characterization can be done by observing spatio temporal behavior of the interface fluctuations. This behavior can be studied within the framework of continuum equations such as a Langevin type equation proposed by Edwards and Wilkinson (EW) [2]. It allows a non-equilibrium statistical mechanical description which brings out the scale invariant nature of the interface roughness. Consequently one obtains two dynamical critical exponents $\alpha$ and $\beta$ (or equivalently $\alpha$ and $z=\alpha / \beta$ ) which completely characterize the growth. The correlation length along the substrate varies as $r^{\alpha}$ and $t^{1 / z}$ whereas that along the normal to the surface exhibits a $t^{\beta}$ behavior.

The geometrical feature of growth represented by the lateral component of the growth velocity was captured by Kardar, Parisi and Zhang (KPZ) [3], by introducing a nonlinear term proportional to $(\nabla h)^{\frac{2}{2}}$ in the EW equation:

$$
\frac{\partial h}{\partial t}=\nu \nabla^{2} h+\frac{\lambda}{2}(\nabla h)^{2}+\eta
$$

where $h(x, t)$ is the height function and $\eta(x, t)$ is a nonconserved gaussian white noise characterized completely by its one and two-point functions:

$$
\begin{aligned}
\langle\eta(\mathbf{x}, t)\rangle & =0 \\
\text { and } \quad\left\langle\eta(\mathbf{x}, t) \eta\left(\mathbf{x}^{\prime}, t^{\prime}\right)\right\rangle & =2 D \delta\left(\mathbf{x}-\mathbf{x}^{\prime}\right) \delta\left(t-t^{\prime}\right)
\end{aligned}
$$

Dynamic renormalization group technique [- 4 applied to the KPZ model yields $z=3 / 2$ and $\alpha=1 / 2$, based on one-loop calculations for a one dimensional substrate. These values of $\alpha$ and $z$ are obtained by looking for a nontrivial fixed point for the set of one-loop flow equations for the parameters $\nu, \lambda$ and $D$ stated above. It turns out that these results are exact in the sense that they hold to all orders and can be obtained by invoking the Galilean invariance of the KPZ equation and the Fokker-Planck equation associated with it for the $1+1$ case [1]. The KPZ equation, however, is of the non-conservative type, (i.e. it can not be written in the form $\frac{\partial h}{\partial t}=\nabla \cdot j$ where $j(\mathbf{x}, t)$ is a local current) and so is relevant mainly for growth with overhangs and vacancies corresponding to the regime of high temperature and high volatility of the deposited material.

A general fourth order conservative equation was proposed by Lai and Das Sarma (LDS) [5] of the form

$$
\begin{gathered}
\frac{\partial h}{\partial t}=\nu_{2} \nabla^{2} h \\
-\nu_{4} \nabla^{4} h+\lambda_{22} \nabla^{2}(\nabla h)^{2} \\
+\lambda_{13} \nabla \cdot(\nabla h)^{3}+\eta
\end{gathered}
$$

If $\lambda_{22}=0$ then in the absence of noise, this equation is symmetric under $h \rightarrow-h$. When $\nu_{2}$ is zero the $\nabla$. $(\nabla h)^{3}$ term is more relevant than the $\nabla^{2}(\nabla h)^{2}$ term as a perturbation to the linear term $\nabla^{4} h$. If one makes the coefficients in this equation time dependent, the situation can change significantly with the possibility of the latter term taking over the former one. Interpretation of this in terms of different relaxation mechanisms operating at different times during the pre-asymptotic region in time will be discussed elsewhere [6].

In a recent paper, Das Sarma and Kotlyar [7], have shown that the most general conserved fourth order nonlinearity, viz. $\lambda_{13} \nabla \cdot(\nabla h)^{3}$ belongs to the universality class of the EW model., i.e. the interaction produces a $-\nu_{2} k^{2}$ term in the inverse propagator at zero frequency, where the coefficient $\nu_{2}$ is proportional to the first power of the perturbation parameter $\lambda_{13}$. This confirmed the earlier numerical evidence based on simulations in $(1+1)$ dimensions due to Kim and Das Sarma 8 .

The purpose of this letter is to show that this analysis is in fact completely general and a general nonlinear conservative growth equation reduces to either Lai-Das Sarma or the EW form. We shall consider the following equation with a general nonlinearity of $\operatorname{order}(2 n+2)$ for integer $n$, 


$$
\begin{aligned}
\frac{\partial h}{\partial t}=\nu \nabla^{2 n+2} h & +\frac{\lambda_{2}}{2 n} \nabla^{2}(\nabla h)^{2 n} \\
& +\frac{\lambda_{1}}{(2 n+1)} \nabla \cdot(\nabla h)^{2 n+1}+\eta
\end{aligned}
$$

Note that the linear term too is of order $(2 n+2)$, in order to keep the nonlinear terms relevant in RG sense.

Consider e.g., the general nonlinearity: $\nabla \cdot(\nabla h)^{2 n+1}$. (The choices $n=0$ and $n=1$ correspond respectively to the EW equation and reference [6]). For the purpose of diagrammatic perturbation theory, it corresponds to a "vertex" with $(2 n+1)$ prongs ending on a cross and one free ended prong.(see, e.g., the book by Barabási and Stanley for notations and conventions). The vertex and the corresponding Feynman rule are in Fig.1. for example $n=1$ it corresponds to the vertex factor of $\frac{1}{3} \lambda_{1}\left(\mathbf{q}_{1} \cdot \mathbf{q}_{2}\right) \mathbf{k} \cdot\left(\mathbf{k}-\mathbf{q}_{1}-\mathbf{q}_{2}\right)$, which upon suitable identification of the parameters and a change of variables becomes the expression of reference [6]. The propagator modification at order $\lambda_{1}$ is shown in Fig.2.

Again, with $n=1$ we recover the one-loop Hartree term of reference [6]. The $n$-loop Hartree diagram shown in figure 2, is equally easy to evaluate; thanks to the peculiar nature of the noise contraction which generates the Feynman factor $2 D \delta\left(\mathbf{k}_{1}+\mathbf{k}_{2}\right) \delta\left(\omega_{1}+\omega_{2}\right)$. The inverse free propagator is given by

$$
G_{0}^{-1}(\mathbf{k}, \omega)=-i \omega+(-1)^{n} \nu k^{2 n+2}
$$

For our purpose (which is to identify the modification of the equation of motion or the hamiltonian whose $h$ variation produces the equation), it is enough to compute the 'vertex function' or the 'amputated' correlation function which amounts to snapping off the external propagators. There is a symmetry factor associated with this diagram given by $\frac{1}{2}(2 n+1) \cdot 2 n(2 n-1)$. Also every noise contraction gives a $2 D$ factor yielding an overall multiplier as $(-1)^{n+1} \lambda_{1} \cdot n(2 n-1)(2 D)^{n}$. Using the standard techniques to evaluate the frequency-momentum integrals, we finally arrive at the amputated $n$-loop Hartree vertex expression as

$$
(-1)^{n+1} \lambda_{1}(D / \nu)^{n} n(2 n-1) K_{d}^{n}\left[\int q^{d-(2 n+1)} d q\right]^{n} \cdot k^{2},
$$

where $K_{d}$ is related to the usual angular integration factor as $K_{d}=\frac{1}{(2 \pi)^{d}} S_{d}$. It is clear from this expression that the $\nabla \cdot(\nabla h)^{2 n+1}$ nonlinearity produces a $\nabla^{2} h$ term (identified as the coefficient of $-k^{2}$ in the above expression) at the same order in $\lambda_{1}$ in dimensions $d<d_{\text {crit }}$. This puts the "odd order" nonlinearities in the EW class.

Exactly similar analysis (see Fig.3 ) of the "even order" nonlinearity $\frac{1}{2 n} \lambda_{2} \nabla^{2}(\nabla h)^{2 n}$ produces the amputated three point function (which is just the object that appears in the equation) given by

$$
k^{2}[\mathbf{q}(\mathbf{k}-\mathbf{q})] \frac{3 \lambda_{2}}{n}\left(\begin{array}{c}
2 n \\
4
\end{array}\right)\left(\frac{D K_{d}}{\nu}\right)^{n-1}\left[\int p^{d-(2 n+1)} d p\right]^{n-1}
$$

Again, we simply identify from this expression that the Lai-Das Sarma term, $\nabla^{2}(\nabla h)^{2}$ is produced at the first order in $\lambda_{2}$ in dimensions less than the critical dimension, putting the even order nonlinearities in the Lai-Das Sarma class.

These arguments are trivially extendible to the nonlinearities of the form $\nabla^{2 m+1} \cdot(\nabla h)^{2 n+1}$ and $\nabla^{2 m}(\nabla h)^{2 n}$ (with the appropriate linear terms in the growth equation to keep the nonlinearities relevant). They respectively generate the generalized EW and the Lai-Das Sarma terms, viz. $\nabla^{2 m} \nabla^{2} h$ and $\nabla^{2 m}(\nabla h)^{2}$ types. Generally a nonlinearity of the kind $\nabla^{2}\left[\nabla^{2 m} h\right]^{2 n}$ collapses to $\nabla^{2}\left(\nabla^{2 m} h\right)^{2}$ and $\nabla \cdot\left[\nabla^{2 m+1} h\right]^{2 n+1}$ to $\nabla^{2}\left[\nabla^{2 m} h\right]$.

The above mechanism of generation of the lower order terms via higher order nonlinearities reflects onto a simple physically meaningful geometric picture of the evolving growth front. Consider e.g., the equation

$$
\frac{\partial h}{\partial t}=\nu \nabla^{2 n+2} h+\lambda_{1} \nabla \cdot(\nabla h)^{2 n+1}+\eta
$$

The rate $\frac{\partial h}{\partial t}$ depends upon the variation of $(\nabla h)^{2 n+1}$, including its sign and $h$ varies locally to attempt to make this rate vanish for a stable growth mode. Note that the nature of the response for terms $\nabla \cdot(\nabla h)$ and $\nabla \cdot(\nabla h)^{2 n+1}$ is exactly the same except for its magnitude, given any geometrical configuration on the surface for any value of $n$ (i.e. the power of the gradient). This fact seems, according to us, to be the root cause for the generation of the EW term since the surface morphology in these cases must be comparable, considering the compatibility of the responses.

Similar reasoning can be furnished in the case of $\nabla^{2}(\nabla h)^{2 n}$ leading to the $\nabla^{2}(\nabla h)^{2}$ term. Here the only difference is that the growth rate does not depend on the sign of $h$, thus making the LDS term as the lowest order term allowed. This leads to the conclusion that in general the nonlinearities are characterized by the order of the variation of $h$ locally, i.e. slope, curvature, etc..., corresponding to $\nabla h, \nabla^{2} h, \cdots$ etc.. and thus a general nonlinearity is of the form $\nabla^{2 m}\left(\nabla^{n} h\right)^{2}$.

\section{ACKNOWLEDGMENTS}

The authors would like to thank Professor S. Das Sarma for a critical reading of the manuscript, valuable suggestions and many insightful remarks which contributed significantly to the improvement of this manuscript. One of us (AKK) would also like to thank Professor Martin Grant for useful correspondence and the 
Department of Physics, University of Pune for hospitality during a long term visit in which the above work was completed.

Electronic Mail:

abhi@rri.ernet.in, abhi@iucaa.ernet.in (AKK), elec@physics.unipune.ernet.in (SVG),

[1] A.L. Barabasi and H.E. Stanley, Fractal Concepts in Surface Growth, Cambridge University Press, N.Y. (1995); Dynamics of Fractal Surfaces, ed. F. Family and T. Vicsek, World Scientific, Singapore (1991); J. Krug and H. Spohn in Solids Far from Equilibrium : Growth, Morphology and Defects, ed. C. Godreche, Cambridge University Press, N.Y. (1991) and references therein.

[2] S.F. Edwards and D.R. Wilkinson, Proc. Royal Soc., London A381, 17 (1982).

[3] M. Kardar, G. Parisi and Y.-C. Zhang, Phys. Rev. Lett. 58, 2087 (1987).

[4] D. Forster, D.R. Nelson and J. Stephen, Phys. Rev. A16, 732 (1977).

[5] Z.W. Lai and S. Das Sarma, Phys. Rev. Lett. 66, 2348 (1991).

[6] S.V. Ghaisas private communications.

[7] S. Das Sarma and R. Kotlyar, Phys. Rev. E50, R4275 (1994)

[8] J.M. Kim and S. Das Sarma Phys. Rev. E51,1889 (1995).

FIG. 1. The vertex for $\nabla \cdot(\nabla h)^{2 n+1}$ nonlinearity. The associated Feynman rule is:

$\frac{(-1)^{n+1}}{(2 n+1)} \lambda_{1}\left(\mathbf{q}_{1} \cdot \mathbf{q}_{2}\right) \cdots\left(\mathbf{q}_{2 n-1} \cdot \mathbf{q}_{2 n}\right)\left[\mathbf{k} \cdot \mathbf{q}_{2 n+1}\right] \delta(\mathbf{k}-$ $\left.\sum_{i=1}^{2 n+1} \mathbf{q}_{i}\right) \delta\left(\omega-\sum_{i=1}^{2 n+1} \omega_{i}\right)$

FIG. 2. The $n$-Loop Hartree diagram generating the EW term with a coefficient proportional to $\lambda_{1}$. Also shown is the two-point vertex function.

FIG. 3. The diagram for the nonlinearity of the type $\nabla^{2}(\nabla h)^{2}$ proportional to $\lambda_{2}$ generated by the nonlinearity $\frac{1}{2 n} \lambda_{2} \nabla^{2}(\nabla h)^{2 n}$. Also shown is the three-point vertex function. 